\section{Surgical Repair of a Sinus of Valsalva Aneurysm Ruptured into the Right Atrium Diag- nosed After a Non-Penetrating Chest Trauma}

\section{Ben Jmaà Hèla ${ }^{1 *}$, Cherif Taieb ${ }^{1}$, Bouassida Abir ${ }^{1}$, Dammak Aiman', Dhouib Faten', Ghorbel Nesrine ${ }^{1}$, Souissi Iheb ${ }^{2}$, Abid Dorra $^{3}$, Elleuch Nizar ${ }^{1}$, Masmoudi Sayda ${ }^{1}$ and Frikha Imed ${ }^{1}$}

${ }^{1}$ Department of Cardiovascular and Thoracic Surgery, Habib Bourguiba Hospital, Sfax, Tunisia

${ }^{2}$ Department of Anesthesiology, Habib Bourguiba Hospital, Sfax, Tunisia

${ }^{3}$ Department of Cardiology, Hedi Chaker Hospital, Sfax, Tunisia

\begin{abstract}
Sinus of Valsalva ruptured aneurysm into right cardiac chambers is an infrequent pathology. It can be congenital or acquired.

In this case report, we describe a case of a ruptured aneurysm of the sinus of Valsalva ruptured into the right atrium discovered incidentally after a non-penetrating chest trauma in a 37 -year-old patient.

The patient underwent surgical closure of the fistula with a synthetic patch under cardiopulmonary bypass without complications.

Keywords: Aneurysm; Fistula; Right atrium; Rupture Sinus of Valsalva; Surgery
\end{abstract}

\section{Introduction}

Aorto-right atrial fistulas are lesions that result in heart failure if they are untreated. They result from rupture of congenital or acquired aortic aneurysms into the right atrium.

We report an unusual case of fistula from aneurysmal right sinus of Valsalva to the right atrium discovered after a non-penetrating trauma and treated surgically.

\section{Case Presentation}

A 37 year-old-man, with no past medical history, was a victim of a closed chest trauma by falling in a hole whose depth was 20 meters.

*Corresponding author: Ben Jmaà Hèla, Department of Cardiovascular and Thoracic Surgery, Habib Bourguiba Hospital, Sfax 3029, Tunisia, Tel: +216 96704740; E-mail: bjhela@yahoo.fr

Citation: Hela BJ, Taieb C, Abir B, Aiman D, Faten D, et al. (2016) Surgical Repair of a Sinus of Valsalva Aneurysm Ruptured into the Right Atrium Diagnosed After a Non-Penetrating Chest Trauma. J Emerg Med Trauma Surg Care 3: 012.

Received: July 08, 2015; Accepted: December 30, 2015; Published: January 14,2016
He was admitted to the emergency department with stable vital signs. He had mandibular fractures. Cerebral CT scan showed no anomalies. Systolic blood pressure was $131 \mathrm{~mm} \mathrm{Hg}$, diastolic blood pressure was $40 \mathrm{~mm} \mathrm{Hg}$. The patient had dyspnea. There was an ecchymosis on the chest wall. A thrill was appreciated over the precordium.

Heart auscultation revealed a continuous murmur on the aortic site. A chest roentgenogram revealed an increased cardiac silhouette with bilateral alveolar opacities. There was a sinus tachycardia on the electrocardiogram, without ischemic signs or conduction disorders. A two-dimensional echocardiography revealed an important left to right shunt with a continuous flow from the aorta to the right atrium, with an aneurysm of the right sinus of Valsalva. The aneurysm was next to the right coronary artery. The aortic valve was without anomalies, and the right heart chambers were dilated. The echocardiography showed no other congenital heart diseases.

Thoracic CT scan detected a bilateral pulmonary edema with a mild pleural effusion. So, the patient underwent emergent surgical repair. A median sternotomy was performed. The pericardium was opened. A continuous thrill along the aorta was palpated.

Cardiopulmonary bypass was established between the aorta and two vena cavae. The intra-operative examination didn't find any hematoma around the sinus of Valsalva which was dilated. A fistula between the right sinus of Valsalva and the right atrium at the level of the right coronary artery was observed through a transverse aortotomy. This defect had $2 \mathrm{~cm}$ of diameter. There was no injury of the aortic valve. The defect was closed with a synthetic patch sutured with pledgeted sutures from inside the right coronary sinus at the level of the right coronary artery into the atrial wall (Figure 1).

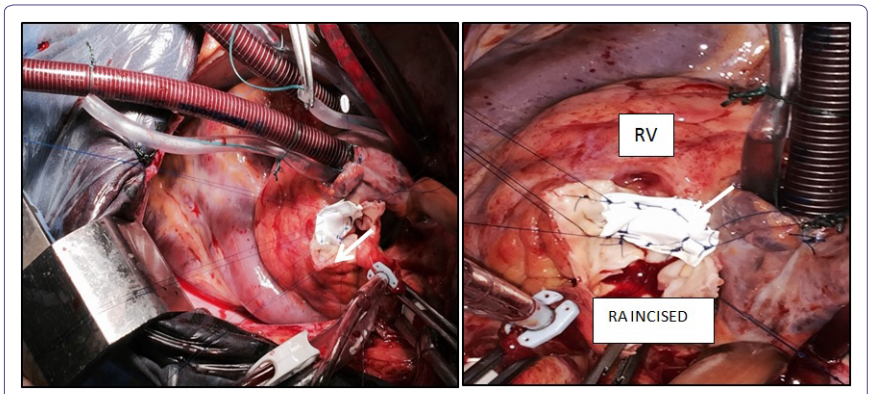

Figure 1: Closure of the fistula between the sinus of Valsalva and the right atrium with a synthetic patch (arrow).

RA: Right Atrium; RV: Right Ventricle

After surgery, the patient's recovery was uneventful and the patient was discharged on the fifth postoperative day. The patient remains asymptomatic and without murmurs 2 months after the surgery.

\section{Discussion}

Aneurysmal dilatation of the sinus of Valsalva is a cardiac anomaly caused by infective endocarditis, syphilitic aortitis, atherosclerosis, connective tissue disease or as a congenital abnormality discovered incidentally $[1,2]$.

Rupture of sinus of Valsalva aneurysm into the cardiac chambers is a complication which predominantly affects males $[3,4]$. When 
aneurysms arise in the right coronary sinus, they mostly communicate with the right atrium, as was the case of our patient [3].

The most notable presenting feature of ruptured sinus aneurysm is congestive heart failure with a left to right shunt [5]. The most common clinical signs are dyspnea, pulmonary edema, with continuous murmur. It can also be asymptomatic and discovered incidentally. The clinical presentation depends on the fistula size and the pressure and flow difference between communicating structures.

The diagnosis is performed by two-dimensional trans-thoracic and trans-esophageal echocardiography, which demonstrates the aneurysmal dilatation with abnormal flow through the ruptured segment and are very helpful for the surgeon $[3,6]$. Three dimensional flow visualization determines the precise location of the fistula [7].

The CT-scan and cardiac MRI are two non-invasive image modalities that can provide important anatomical and functional information and allow exact description of the lesion and help to planning of cardiac surgery [8,9].

This case represents a fistulous connection between the aneurysmal sinus of Valsalva and the right atrium that was unrecognized, and was discovered after a non-penetrating chest trauma in an adult, and describes surgical technique of closure.

M Chessa et al., [10] described a case of congenital fistula between the right sinus of Valsalva and the right atrium without dilatation of the sinus of Valsalva in an asymptomatic adult. This fistula was sutured at its origin in the aortic sinus and at its termination in the right atrium.

In our case, the diagnosis of the asymptomatic fistula was suspected by clinical examination after chest trauma which showed a precordial continuous murmur, and confirmed by tans-thoracic echocardiography. The etiology may be congenital. It was closed surgically with a prosthetic patch through a transverse aortotomy.

\section{Conclusion}

Aorto-atrial fistula is a fistulous communication that may present as an incidental finding or with heart failure with pulmonary edema.
Surgical repair is the treatment of choice. We described a rare case of aneurysmal right sinus of Valsalva ruptured into the right atrium in adult patient revealed by a chest trauma. A successful surgical closure of the fistula was undergone.

\section{References}

1. Guo DW, Cheng TO, Lin ML, Gu ZQ (1987) Aneurysm of the sinus of Valsalva: a roentgenologic study of 105 Chinese patients. Am Heart J 114: 11691177.

2. Feldman DN, Roman MJ (2006) Aneurysms of the sinuses of Valsalva. Cardiology 106: 73-81.

3. Missault L, Callens B, Taeymans Y (1995) Echocardiography of sinus of Valsalva aneurysm with rupture into the right atrium. Int J Cardiol 47: 269-272.

4. Walpot J, Klazen C, Hokken R, Sorgedrager J, Hoevenaar M, et al. (2005) Aorto-right ventricular fistula as an occasional finding. Eur J Echocardiogr 6: 65-66.

5. Gibbs KL, Reardon MJ, Strickman NE, de Castro CM, Gerard JA, et al. (1986) Hemodynamic compromise (tricuspid stenosis and insufficiency) caused by an unruptured aneurysm of the sinus of Valsalva. J Am Coll Cardiol 7: 11771181.

6. Rubin DC, Carliner NH, Salter DR, Plotnick GD, Hawke MW (1992) Unruptured sinus of Valsalva aneurysm diagnosed by transesophageal echocardiography. Am Heart J 124: 225-227.

7. Thakrar D, Popescu A, Gupta S, de Freitas A, Russell H, et al. (2013) Complex 3D blood flow pathways in two cases of aorta to right heart fistulae: a 4D flow MRI study. Magn Reson Imaging 31: 1453-1455.

8. Siebers C, Schrammb R, Friedmanna A, Weig T (2014) Severe cardiogenic shock due to acute onset of an aorto-to-right atrial shunt in a patient with aortic valve endocarditis. International Journal of Surgery Case Reports 5 : 108-110.

9. Azarine A, Lions C, Koussa M, Beregi JP (2001) Rupture of an aneurysm of the coronary sinus of Valsalva: diagnosis by helical CT angiography. Eur Radiol 11: 1371-1373.

10. Chessa M, De Rosa G, Giamberti A, Butera G, Carminati M (2006) Congenital aortico-right atrial communication: a rare case in an adult patient. Int $\mathrm{J}$ Cardiol 113: 105-106 